\title{
UCSF
}

UC San Francisco Previously Published Works

Title

Implementing pediatric inpatient asthma pathways.

Permalink

https://escholarship.org/uc/item/8674c074

Journal

The Journal of asthma : official journal of the Association for the Care of Asthma, 58(7)

ISSN

0277-0903

Authors

Gupta, Nisha

Cattamanchi, Adithya

Cabana, Michael D

et al.

Publication Date

2021-07-01

DOI

10.1080/02770903.2020.1741612

Peer reviewed 


\title{
Implementing pediatric inpatient asthma pathways
}

\author{
Nisha Gupta, Adithya Cattamanchi, Michael D. Cabana, Brittany Jennings, \\ Kavita Parikh \& Sunitha V. Kaiser
}

To cite this article: Nisha Gupta, Adithya Cattamanchi, Michael D. Cabana, Brittany Jennings, Kavita Parikh \& Sunitha V. Kaiser (2020): Implementing pediatric inpatient asthma pathways, Journal of Asthma, DOI: 10.1080/02770903.2020.1741612

To link to this article: https://doi.org/10.1080/02770903.2020.1741612

View supplementary material ¿

Accepted author version posted online: 11

Mar 2020.

Published online: 18 Mar 2020.

Submit your article to this journal ${ }^{\top}$

Џ Article views: 69

View related articles $ک \pi$

View Crossmark data $\asymp$ 


\title{
Implementing pediatric inpatient asthma pathways
}

\author{
Nisha Gupta, MD ${ }^{a}$, Adithya Cattamanchi, MD, MAS ${ }^{b}$, Michael D. Cabana, MD, MPH ${ }^{a, c}$, Brittany Jennings, MHA ${ }^{d}$, \\ Kavita Parikh, MD, MSHS ${ }^{\mathrm{e}}$, and Sunitha V. Kaiser, MD, MSc ${ }^{\mathrm{a}}$ \\ ${ }^{a}$ Department of Pediatrics, University of California, San Francisco, CA, USA; ${ }^{b}$ Department of Medicine, University of California, San \\ Francisco, CA, USA; 'Pediatrics, Philip R. Lee Institute for Health Policy Studies University of California, San Francisco, CA, USA;

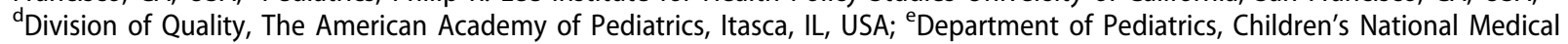 \\ Center, Washington, DC, USA
}

\begin{abstract}
Objective: Pathways are succinct, operational versions of evidence-based guidelines. Studies have demonstrated pathways improve quality of care for children hospitalized with asthma, but we have limited information on other key factors to guide hospital leaders and clinicians in pathway implementation efforts. Our objective was to evaluate the adoption, implementation, and reach of inpatient pediatric asthma pathways.

Methods: This was a mixed-methods study of hospitals participating in a national collaborative to implement pathways. Data sources included electronic surveys of implementation leaders and staff, field observations, and chart review of children ages 2-17 years admitted with a primary diagnosis of asthma. Outcomes included adoption by hospitals, pathway implementation factors, and reach of pathways to children hospitalized with asthma. Quantitative data were analyzed using descriptive statistics and multivariable regression. Qualitative data were analyzed using thematic content analysis.

Results: Eighty-five hospitals enrolled; 68 (80\%) adopted/completed the collaborative. These 68 hospitals implemented pathways with overall high fidelity, implementing a median of 5 of 5 core pathway components (Interquartile Range [IQR] 4-5) in a median of 5 months (IQR 3-9). Implementation teams reported a median time cost of $78 \mathrm{~h}$ (IQR: 40-120) for implementation. Implementation leaders reported the values of pathway implementation included improvements in care, enhanced interdisciplinary collaboration, and access to educational resources. Leaders reported barriers in modifying electronic health records (EHRs), and only $63 \%$ of children had electronic pathway orders placed.

Conclusions: Hospitals implemented pathways with high fidelity. Barriers in modifying EHRs may have limited the reach of pathways to children hospitalized with asthma.
\end{abstract}

Abbreviations: AAP: American Academy of Pediatrics; QI: quality improvement; EHR: electronic health record

\section{ARTICLE HISTORY}

Received 9 December 2019

Revised 24 February 2020

Accepted 6 March 2020

\section{KEYWORDS}

Asthma; child; clinical pathway; quality

improvement

\section{Introduction}

Asthma affects nearly $10 \%$ of American children. Although asthma-related hospitalizations are largely preventable, asthma remains a leading cause of childhood hospitalization (1,2). Pathways are succinct versions of guidelines that visually guide healthcare providers stepby-step through the evidence-based care of children with a specific medical condition. Pathways promote standardization of care, with the aim of improving care quality and decreasing unnecessary healthcare utilization. They have been shown to improve care for children hospitalized with asthma, including increasing use of recommended medications, and decreasing length of hospital stay, healthcare costs, hospital readmissions, and healthcare disparities (3-9).

Most prior studies of pathways have focused on determining clinical effectiveness $(3,5,7,9)$. While such studies are important, they provide limited guidance on the implementation process for those considering or leading pathway implementation. A study by Kaiser et. al. detailed potential barriers and best practices in asthma pathway implementation (10), and a study by Pound et. al. described clinicians' satisfaction with asthma pathway implementation (11). However, both of these studies were limited to children's hospitals. Therefore, prior studies of pathways leave critical

CONTACT Dr. Sunitha Kaiser sunitha.kaiser@ucsf.edu Department of Pediatrics, University of California, 550 16th Street, Box 3214, San Francisco, CA 94158, USA. 
gaps in our understanding of pathways, such as the feasibility, fidelity, and time costs of pathway implementation across diverse hospital settings.

In 2018, the American Academy of Pediatrics (AAP) Value in Inpatient Pediatrics network launched a national learning collaborative to implement pediatric inpatient asthma pathways. Pathway implementation was associated with significant improvements in quality of care, including increases in early use of metered-dose inhalers (MDI) and referral of caregivers to smoking cessation resources. In addition, placement of pathway orders in the electronic health record was associated with an $8 \%$ reduction in length of hospital stay (LOS) (12). The purpose of this study was to evaluate the adoption, implementation, and reach of pediatric inpatient asthma pathways across the diverse group of hospitals from around the United States that participated in this collaborative.

\section{Methods}

\section{Study design, setting, and population}

This was a mixed-methods study of hospitals participating in a national learning collaborative- Pathways for Improving Pediatric Asthma Care (PIPA) (12). PIPA was led by the AAP's Value in Inpatient Pediatrics (VIP) Network, the inpatient pediatric quality improvement (QI) network at the AAP (13). Recruitment occurred via the VIP electronic mailing list (listserv). The listserv includes clinicians from 250 hospitals around the country that range widely in size, type (e.g. children's, community), ownership model (e.g. private, nonprofit), and location (e.g. rural, urban). Hospitals that joined the collaborative were structurally and geographically varied, and the populations of children they serve mirror the diversity of the general U.S. population. To participate in this collaborative, each institution paid $\$ 750$ (to cover administrative costs). The study took place from January 2018-March 2019 and was approved by the AAP institutional review board.

\section{Intervention development}

A panel of experts assembled by the AAP met regularly throughout 2017 to determine project aims and select intervention components that supported these aims (Figure 1). Members of this this panel worked in diverse hospital settings and had expertise in pediatric hospital medicine, pediatric emergency medicine, asthma, quality measurement, quality improvement, and health services research. Additionally, 2 nurses and 1 respiratory therapist reviewed and provided feedback on the intervention.

\section{Intervention description}

Participating hospitals were provided a pediatric asthma pathway implementation toolkit (14), which included sample evidence-based pathways and sample order sets based on pathway content. The five core pathway components included guidance on selection and dosing of medications (e.g. MDI), standardized instructions for titrating bronchodilator therapy based on asthma severity, reminders to screen for secondhand

Specific Aims

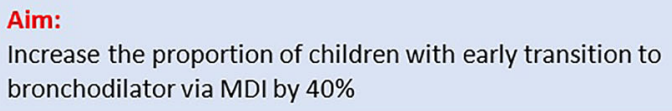

Core Pathway Components
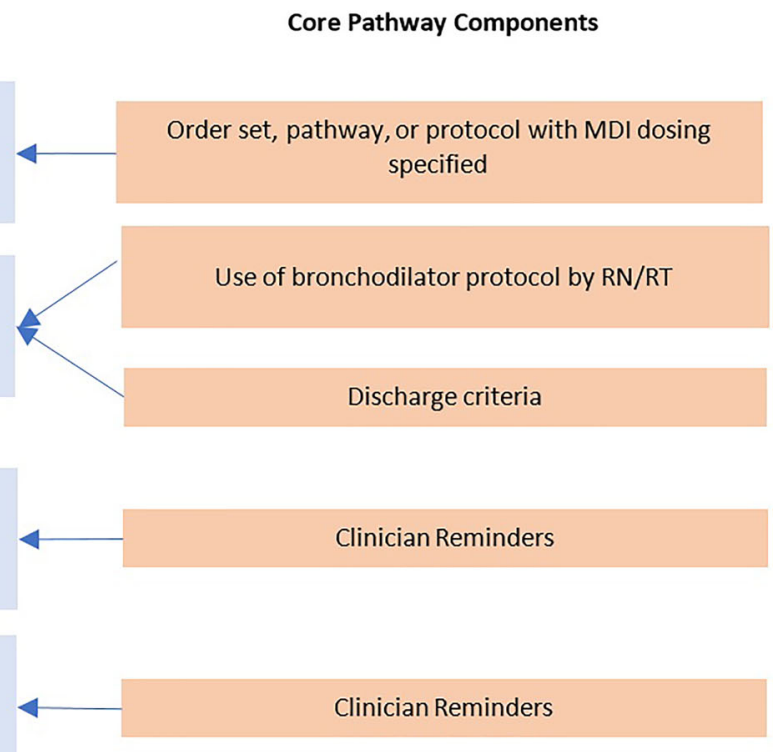

Figure 1. Blue boxes describe specific aims, orange boxes describe core pathway components, and arrows show the relationship between these components and the specific aims. 
tobacco smoke exposure, reminders to refer caretakers to resources for smoking cessation, and hospital discharge criteria. Each hospital designated a local physician implementation leader, all of whom were pediatric hospitalists. These leaders recruited local multidisciplinary teams, including nurses, respiratory therapists, pharmacists, and administrators. Local teams adapted and implemented the pathways to fit local needs and context (e.g. deciding whether nurses or respiratory therapists would oversee bronchodilator titration, determining where to integrate reminders to screen for secondhand tobacco, refining bronchodilator protocol dosing guidance and discharge criteria based on local consensus). Implementation involved educating local clinicians on these core pathway components and integrating these core pathway components into clinical workflows (either via paper visual aids or integration into the electronic health record [EHR]). To support pathway implementation, we used a learning collaborative model (15), and each hospital's physician implementation leader was provided a physician mentor to guide them in the learning collaborative. Mentors were selected based on experience in pediatric hospital medicine, QI, and community hospitals. These mentors met with local implementation leaders monthly via teleconference for 2 months before and 4 months after pathway implementation began to provide support in engaging hospital leadership, garnering local healthcare provider buy-in, planning improvement cycles (15), and addressing institutional barriers. Hospitals were provided several additional resources for implementation support, including educational videoconference sessions, educational materials, QI training, monthly videoconferences for all local implementation leaders to facilitate peer learning, in-person meetings at national conferences, monthly audit and feedback (on local and national performance), and a free mobile application with pathway content. Educational videoconference session topics included: evidence-based asthma care, quality improvement methodology, leadership, and sustaining quality improvements. Educational materials included evidence-based pathways and supporting literature.

\section{Theoretical framework}

We applied the RE-AIM implementation science framework (16) to gain a broad understanding of the impact of the intervention across five domains: reach, effectiveness, adoption, implementation, and maintenance. For this study, we evaluated adoption, implementation, and reach. Effectiveness was evaluated separately and maintenance is currently being evaluated. Table 1 outlines the components of the REAIM framework we evaluated, data collection, and outcomes. Adoption refers to willingness of clinicians to implement an intervention. Implementation refers to the fidelity, or consistency, with which the intervention is delivered and the associated costs of delivering the intervention. Reach assesses the extent to which the target population of patients who should have received the intervention actually received it.

\section{Outcomes}

\section{Adoption}

We evaluated 2 outcomes: 1) participation in the learning collaborative and associated outcome monitoring for the full 15-month study duration, and 2) perceived barriers to fully participating/adopting (elicited with the question: "Please describe what factors that led your hospital withdrawing from participating in the project."). Our hypothesis was that hospitals that participated fully/adopted would not significantly differ from those who dropped out.

\section{Implementation}

We evaluated 5 outcomes: 1) fidelity (the number of core pathway components implemented), 2) time costs (total number of hours spent on implementation by local implementation teams), 3) implementation strategies utilized, 4) perceived value of time spent on

Table 1. RE-AIM Domains, data collection, and outcomes.

\begin{tabular}{|c|c|c|c|}
\hline RE-AIM Domain & Description & Data Collection & Outcomes \\
\hline Adoption & $\begin{array}{l}\text { Willingness of clinicians to } \\
\text { implement an intervention }\end{array}$ & $\begin{array}{l}\text { Physician implementation leader } \\
\text { and staff surveys }\end{array}$ & $\begin{array}{l}\text { - Participation in the collaborative and associated data } \\
\text { monitoring for the full study duration } \\
\text { - Perceived barriers to adoption }\end{array}$ \\
\hline Implementation & $\begin{array}{l}\text { Consistency with which an } \\
\text { intervention is delivered (fidelity) } \\
\text { and the associated costs of } \\
\text { delivering the intervention }\end{array}$ & $\begin{array}{l}\text { Physician implementation leader } \\
\text { surveys and field observations }\end{array}$ & $\begin{array}{l}\text { - } \quad \text { Fidelity - Number of core components implemented } \\
\text { - Time costs - Total number of hours spent on } \\
\text { implementation by implementation teams } \\
\text { - Implementation strategies utilized } \\
\text { - Perceived value of time spent on pathway } \\
\text { - } \quad \text { Perceived barriers to pathway implementation }\end{array}$ \\
\hline Reach & $\begin{array}{l}\text { Extent to which the intervention } \\
\text { reached the target population }\end{array}$ & Chart review & $\begin{array}{l}\text { - Placement of an electronic pathway/protocol order for } \\
\text { standardized bronchodilator titration }\end{array}$ \\
\hline
\end{tabular}


implementation, and 5) perceived barriers to pathway implementation. Our hypothesis was that participating hospitals would achieve high fidelity implementation.

\section{Reach}

Developing electronic orders for standardized titration of bronchodilators has been described as a key element of pathway implementation $(10,17)$. We evaluated 1 outcome for "Reach," placement of an electronic order in a patient's chart to use a pathway/protocol for standardized bronchodilator titration. This outcome was selected because it is a direct measure of pathway utilization at the patient level. Our hypothesis was that the majority of children would be reached by the pathway intervention.

\section{Data collection}

Data collection occurred in three forms: 1) electronic surveys at all participating hospitals, 2) chart review of children admitted with asthma at all participating hospitals, and 3) field observations of 4 participating hospitals. All survey instruments were beta-tested by a group of 10 pediatric hospitalist physicians. Each hospital's physician implementation leader was surveyed at the beginning of the study to collect information on hospital characteristics. These responses were verified using data from the American Hospital Association Annual Survey Database (18). Data on organizational readiness to change were collected via electronic surveys of staff members at each hospital who were involved in the care of children with asthma (e.g.physicians, nurses, respiratory therapists; range of 2-20 survey responses per hospital). These surveys were designed based on the organizational readiness to change assessment tool (19) and summary scores were calculated for each hospital (range 1-5). Surveys on the implementation process (Supplementary Appendix) were distributed to physician implementation leaders monthly throughout the pathway implementation period (January 2018-March 2019) and at the end of the implementation period (final survey in March 2019). Any discrepancies in survey responses on the implementation process were resolved via direct contact with physician implementation leaders at the end of the study.

Data collection also involved chart review of children 2-17 years old hospitalized with a primary diagnosis of asthma. Chart review was performed at each site by local physicians or nurses. Reviewers were provided chart review manuals and trained via videoconference by the central research team. Charts from January 2018-March 2019 were reviewed prospectively (during and after pathway implementation). Charts were selected in chronologic order each month (all admissions up to a max of 20 per month per hospital). Charts were excluded if children had chronic medical conditions that precluded pathway use. These included chronic lung disease (e.g. cystic fibrosis, restrictive lung disease, bronchopulmonary dysplasia), congenital or acquired heart disease, airway issues (e.g.vocal cord paralysis, tracheomalacia, tracheostomy dependence), immune disorders, sickle cell anemia, or neuromuscular disorders.

A field observation guide was developed and reviewed by our study group. In April 2019, one author (N.G.) conducted the field observations at 4 study sites within geographic proximity to the central research team (3 community hospitals and 1 free-standing children's hospital). During field observations, self-reported survey data from physician implementation leaders was verified and additional qualitative data on barriers to implementation was collected.

Both chart review and survey administration were done through REDCap 8.5 (Nashville, TN). Data quality was audited monthly by the central research team and by quality audit functions within REDCap.

\section{Analyses}

\section{Adoption}

We compared characteristics of those hospitals that "adopted" (defined as participating in the learning collaborative and associated outcome monitoring for the full study duration) to those hospitals that dropped out of the collaborative using descriptive statistics and univariate tests (Chi-square test for categorical variables, Student's t-test or Mann-Whitney $U$ test for interval variables). Free-text data on barriers to adoption were summarized by frequency of occurrence.

\section{Implementation}

Data on fidelity, time costs, and implementation strategies (e.g. educational videoconferences, improvement cycles, staff training) were summarized using descriptive statistics. A staff training score was calculated for each hospital based on the proportion of clinicians (e.g. physicians, nurses, respiratory therapists) trained in the use of each of the 5 core pathway components (for each component: 0 points if no providers trained, 1 point if some providers trained, 2 points if all providers trained; max score of 10 for 5 core components). We compared implementation data by hospital type (children's versus community hospitals) using univariate tests (Chi-square 
test for categorical variables, Student's t-test or MannWhitney $U$ test for interval variables).

Qualitative data on perceived value of implementation and barriers to implementation were collected via 2 free-text questions (multiple responses allowed):

"Did you find the PIPA project a valuable use of your and hospital staff's time? Please explain why or give an example of how the project was valuable/ not valuable."

"Why was this PIPA core intervention not implemented?"

These qualitative data and notes from field observations were analyzed using thematic content analysis. Two authors (N.G. and S.K.) independently coded the data and developed a preliminary codebook. These two authors then developed consensus on the codebook and descriptions of common themes. Differences were resolved through iterative discussions informed by review of the collected data and development of consensus across all authors.

\section{Reach}

We determined the proportion of children that had electronic pathway orders placed. This analysis only included: 1) hospitals that completed the collaborative, and 2) admissions that occurred during/after pathway implementation (January 2018 to March 2019). We used a multi-level logistic regression model (levels: patient, hospital) to analyze patient characteristics associated with an increased likelihood of a pathway order being placed. These characteristics included age, sex, prior prescription of an inhaled corticosteroid (proxy for chronic asthma severity), and insurance type (proxy for socioeconomic status).

All quantitative analyses were performed with SAS 9.4 (Cary, NC), and p values less than 0.05 were considered statistically significant. All qualitative analyses were performed with Atlas.ti version 7 (Berlin, Germany).

\section{Results}

\section{Adoption}

Physicians from a total of 250 hospitals were contacted via email and 85 hospitals (34\%) enrolled in the learning collaborative. Hospitals that joined were diverse in terms of size, type, and location (Table 2). Most (92\%) were teaching hospitals. Of the 85 hospitals that joined, $68(80 \%)$ participated in the learning collaborative and associated outcome monitoring for the full study duration. Table 2 describes the characteristics of hospitals that completed the collaborative versus hospitals that dropped out. No hospital characteristics were statistically significantly associated with dropping out.

We contacted physician implementation leaders (pediatric hospitalists) from the 17 hospitals that dropped out, and 9 (53\%) provided responses describing the reasons for drop out. Reasons included (in order of frequency): lack of support from hospital leadership/administrators $(n=3)$, difficulty obtaining local institutional review board (IRB) approval $(n=2)$, difficulty obtaining chart review data $(n=1)$, staff turnover $(n=1)$, difficulty due to competing QI projects $(n=1)$, and very low patient volumes $(n=1)$.

\section{Implementation}

Physician implementation leaders from the 68 hospitals that completed the collaborative were surveyed regarding implementation, and all responded (Table 3).

\section{Fidelity}

Hospitals were able to successfully implement the 5 core components of the pathway intervention (median 5 components, Interquartile Range [IQR] 4-5 components). It took a median of 5 months to complete pathway implementation (IQR 3-9 months). A total of 39 hospitals (57\%) were able to integrate pathways into the EHR. These outcomes did not vary significantly by hospital type.

During field observations at 4 participating hospitals, we verified physician implementation leaders' survey responses regarding implementation of core intervention components. All 4 hospitals visited had self-reported implementation of all 5 core pathway components, and only 1 component at 1 hospital site was not visually verified (total 19 of 20 survey responses/core pathway components verified).

\section{Time costs}

The median total time spent on implementation by local implementation teams over the 15-month collaborative was $78 \mathrm{~h}$ (IQR $40-120 \mathrm{~h}$ ). This did not vary significantly by hospital type.

All respondents $(68 / 68,100 \%)$ also provided freetext survey responses on perceived value of and barriers to implementation. Descriptions of the most common themes with exemplary quotes are presented below in order of frequency of occurrence.

\section{Perceived Value of Implementation}

Theme 1: Implementation leaders frequently reported that they valued the improvements in quality of care for children that they experienced with pathway implementation. Implementation leaders reported 
Table 2. Characteristics of Hospitals that Completed versus Dropped Out of the PIPA Collaborative.

\begin{tabular}{|c|c|c|c|c|}
\hline & $\begin{array}{l}\text { All hospitals } \\
\quad(n=85)\end{array}$ & $\begin{array}{l}\text { Hospitals that Completed } \\
\text { PIPA Collaborative } \\
(n=68)\end{array}$ & $\begin{array}{l}\text { Hospitals that Dropped Out } \\
\qquad(n=17)\end{array}$ & $p$-value \\
\hline \multicolumn{5}{|l|}{ Hospital Type $(n, \%)$} \\
\hline Children's Hospital & $40(47.1 \%)$ & $34(50 \%)$ & $6(35.3 \%)$ & 0.28 \\
\hline Community Hospital & 45 (52.9\%) & $34(50 \%)$ & $11(64.7 \%)$ & \\
\hline Teaching Hospital $(n, \%)$ & 78 (91.8\%) & $62(91.2 \%)$ & $16(94.1 \%)$ & 0.69 \\
\hline $\begin{array}{l}\text { Inpatient Pediatric Beds } \\
\text { (mean, SD) }\end{array}$ & $39.3(50.9)$ & $42.1(55.2)$ & $28.4(26.6)$ & 0.32 \\
\hline Hospital size $(n, \%)$ & & & & 0.42 \\
\hline Small $(<100)$ & $6(7.1 \%)$ & $6(8.8 \%)$ & $0(0.0 \%)$ & \\
\hline Medium (100-249) & $25(29.4 \%)$ & 19 (27.9\%) & $6(35.3 \%)$ & \\
\hline Large $(\geq 250)$ & $53(62.4 \%)$ & $42(61.8 \%)$ & $11(64.7 \%)$ & \\
\hline Geographic Region $(n, \%)$ & & & & 0.77 \\
\hline West & $18(21.2 \%)$ & $15(22.1 \%)$ & $3(17.6 \%)$ & \\
\hline South & $26(30.6 \%)$ & 19 (27.9\%) & $7(41.2 \%)$ & \\
\hline Northeast & $14(16.5 \%)$ & $11(16.2 \%)$ & $3(17.6 \%)$ & \\
\hline Midwest & $27(31.8 \%)$ & $23(33.8 \%)$ & $4(23.5 \%)$ & \\
\hline Location $(n, \%)$ & & & & 0.08 \\
\hline Urban & 39 (45.9\%) & $32(47.1 \%)$ & 7 (41.2\%) & \\
\hline Suburban & $38(44.7 \%)$ & $32(47.1 \%)$ & $6(35.3 \%)$ & \\
\hline Rural & $8(9.4 \%)$ & $4(5.9 \%)$ & $4(23.5 \%)$ & \\
\hline Presence of PICU $(n, \%)$ & $52(61.2 \%)$ & $44(64.7 \%)$ & $8(47.1 \%)$ & 0.18 \\
\hline Ownership Model $(n, \%)$ & & & & 0.09 \\
\hline Government & $9(10.6 \%)$ & $6(8.8 \%)$ & $3(17.6 \%)$ & \\
\hline Private, nonprofit & $68(80 \%)$ & $57(83.8 \%)$ & $11(64.7 \%)$ & \\
\hline Private, investor owned & $6(7.1 \%)$ & $3(4.4 \%)$ & $3(17.6 \%)$ & \\
\hline $\begin{array}{l}\text { Inclusion in Hospital } \\
\text { Network }(n, \%)\end{array}$ & $65(76.5 \%)$ & $53(77.9 \%)$ & $12(70.6 \%)$ & 0.45 \\
\hline $\begin{array}{l}\text { Presence of Electronic } \\
\text { Health Record }(n, \%)\end{array}$ & $83(97.6 \%)$ & $66(97.1 \%)$ & 17 (100\%) & 0.47 \\
\hline $\begin{array}{l}\text { Total Physicians on Staff for } \\
\text { Inpatient Pediatric Unit } \\
\text { (mean, SD) }\end{array}$ & $7.61(6.4)$ & $7.96(6.9)$ & $6.23(3.4)$ & 0.32 \\
\hline $\begin{array}{l}\text { Leadership Support } \\
\text { Obtained Prior to } \\
\text { Initiation }(n, \%)\end{array}$ & $78(91.8 \%)$ & $63(92.6 \%)$ & $15(88.2 \%)$ & 0.88 \\
\hline $\begin{array}{l}\text { Prior Participation in a } \\
\text { National Learning } \\
\text { Collaborative }(n, \%)\end{array}$ & $57(67.1 \%)$ & 47 (92.6\%) & $10(58.8 \%)$ & 0.73 \\
\hline $\begin{array}{l}\text { Organizational Readiness to } \\
\text { Change Score (mean, SD) }\end{array}$ & $4.09(0.2)$ & $4.08(0.2)$ & $4.18(0.2)$ & 0.22 \\
\hline
\end{tabular}

Table 3. Differences in pathway implementation by hospital type.

\begin{tabular}{|c|c|c|c|c|}
\hline & All hospitals $(n=68)$ & $\begin{array}{l}\text { Children's Hospital } \\
\qquad(n=34)\end{array}$ & $\begin{array}{l}\text { Community Hospital } \\
\qquad(n=34)\end{array}$ & $p$-value \\
\hline Core Interventions Implemented [max: 5] (median, IQR) & $5(4,5)$ & $5(4,5)$ & $5(4.5,5)$ & 0.49 \\
\hline Months Taken to Complete Implementation (median, IQR) & $5(3,9)$ & $5(4,10)$ & $4(3,8)$ & 0.15 \\
\hline $\begin{array}{l}\text { Total Hours Local Leaders Spent on Implementation } \\
\text { (median, IQR) (median, IQR) }\end{array}$ & $78(40,120)$ & $98(55,135)$ & $55(30,110)$ & 0.26 \\
\hline Electronic Health Record Integration ( $n, \%)$ & $39(57 \%)$ & $22(65 \%)$ & $17(50 \%)$ & 0.22 \\
\hline Educational Webinars Attended [max: 7] (median, IQR) & $5(4,7)$ & $5(4,6)$ & $5.5(4,7)$ & 0.95 \\
\hline Staff Training* [max: 10] (median, IQR) & $7(4,10)$ & $5(4,8)$ & $9(5,10)$ & 0.20 \\
\hline Improvement Cycles Completed (median, IQR) & $3(2,5)$ & $4(2,5)$ & $3(2,4)$ & 0.23 \\
\hline
\end{tabular}

The 68 hospitals that completed the collaborative were included in the analyses of implementation.

*Staff training score calculated based on the proportion of clinicians (e.g., physicians, RTs, nursing, and other support staff) trained in core interventions

improvements in outcomes for children with asthma (e.g., decreases in length of hospital stay) as well as spillover effects to children with other conditions, such as bronchiolitis.

"The PIPA project allowed us to enact an asthma pathway, which allows our RTs [respiratory therapists] and nurses more autonomy, makes our asthma care more efficient, and hopefully decreases length of stay. Probably, the biggest change I saw was a sharp increase in MDI [metered dose inhaler] use as compared to nebulizer use." (Site 82)

"The inpatient PIPA project was a valuable us[e] of our hospital's time. We were able to transition to MDIs effectively. We also improved our screening for tobacco exposure and our counseling for the patients." (Site 18) 
Table 4. Characteristics of children for whom pathways were ordered versus not ordered.

\begin{tabular}{llll}
\hline Patient Characteristics & Pathway Order $(n=3502)$ & No Pathway Order $(n=2055)$ & $p$-value \\
\hline Male $(n, \%)$ & $2120(60.5 \%)$ & $1239(60.3 \%)$ & 0.27 \\
Age in years (median, IQR) & $5(3,9)$ & $5(3,9)$ & 0.59 \\
Prior Inhaled Corticosteroid Use $(n, \%)$ & $1760(50.3 \%)$ & $998(48.6 \%)$ & 0.97 \\
Insurance* $(n, \%)$ & & 0.03 \\
$\quad$ Private & $2102(60 \%)$ & $1429(69.5 \%)$ & $532(25.9 \%)$ \\
Public & $1048(29.9 \%)$ & $15(1.1 \%)$ & $62(3.0 \%)$ \\
Tri-care & $47(1.1 \%)$ & $190(5.4 \%)$ & \\
Other, Self-pay, or Unknown & $190)$ \\
*Insurance was used as a proxy for socioeconomic status. Public insurance is provided for children whose families earn less than $133 \%$ \\
of the federal poverty level (20). Tri-care insurance is provided for children of families with members serving in the U.S. military.
\end{tabular}

Theme 2: Access to educational videoconferences and materials was often reported as a valuable part of implementation and the collaborative.

"Yes (it was valuable). Having an organized project with supporting evidence and education allowed for much more efficient implementation and getting providers and nursing staff buy-in in a timely manner." (Site 20)

"AAP provided valuable education through educational videoconferences on quality improvement tools, design and utilization of Plan Do Study Act cycles... and strategies to overcome challenges and promote sustainability." (Site 69)

Theme 3: Implementation leaders also described how they valued the cultivation of interdisciplinary collaboration. They described increased collaboration among physicians across varying specialties, nurses, respiratory therapists, and pharmacists.

"It allowed for a collaborative partnership across multiple healthcare settings (inpatient, ED, and even PICU) while bringing in the expertise of various healthcare disciplines to standardize processes including albuterol titration via pathway, albuterol nebulizer dosing, and steroid administration." (Site 14)

"We wanted to make sure that we were providing the best possible care for our asthma patients through collaboration with ED, inpatient, pharmacy, respiratory and nurses." (Site 37)

\section{Barriers to implementation}

Theme 1: Implementation leaders described difficulties in modifying electronic health records as a common barrier to implementation.

"We initiated the process, but need to obtain approval and buy-in from 5 affiliated hospitals in order to update the inpatient pathway and change the order set and respiratory score in the EHR." (Site 83)

"Other [EHR] builds have taken priority thus we do not have a specific order set in place." (Site 52)

Theme 2: Implementation leaders also reported difficulty obtaining consensus on practice changes as a barrier to implementation. This especially applied to changing the scope of practice of nurses and/or respiratory therapists in autonomously titrating albuterol per pathway recommendations.

\begin{abstract}
"[There were] many institutional barriers to implementing new processes that involve nurses performing additional tasks or making 'clinical decisions' such as spacing albuterol, which were viewed as outside the scope of practice by some." (Site 66)

"MDs were not comfortable that the RNs were scoring patients correctly, and therefore uncomfortable with them weaning patients." (Site 12)
\end{abstract}

\section{Reach}

Among the 5557 patients cared for after pathway implementation, 3502 (63\%) had an electronic pathway order placed (Table 4). Rates of placement of pathway orders ranged widely across hospitals, with a median of $73 \%$ and IQR of $20-97 \%$. Pathway order placement did not differ significantly by patient age, sex, or prior inhaled corticosteroid use. Patients with public insurance were more likely to have a pathway order placed than children with private insurance.

\section{Discussion}

Several studies have demonstrated that pathways are effective in improving quality of care and health outcomes for children hospitalized with asthma $(3-9,12)$. However, none, to our knowledge, have detailed the pathway implementation process across a large, diverse group of hospital settings. We found that pathway implementation was feasible across diverse hospital settings, with $80 \%$ adopting/completing the collaborative and successfully completing pathway implementation in a median of 5 months. Implementation leaders found pathway implementation valuable, and the national learning collaborative provided critical supports for improving pediatric asthma care, including educational materials and videoconferences. Implementation leaders found that modifying EHRs posed a significant barrier to improvement efforts, and this may have limited the 
reach of pathways to children hospitalized with asthma. Policymakers, clinicians, and hospital leaders seeking to improve care for hospitalized children can use these findings to guide future pathway implementation efforts.

The $20 \%$ drop out rate we experienced is similar to prior studies of quality collaboratives focused on implementing pediatric evidence-based guidelines $(21,22)$. But our findings illuminate the common barriers that were associated with discontinuing participation. Lack of support from hospital leadership/ administrators was commonly reported. This barrier may be heightened in community hospital settings, which predominantly care for and prioritize resources for adults (23). Early engagement between implementation leaders and hospital administrators to discuss the goals and potential benefits of pathway implementation may help overcome this barrier (10).

Physician implementation leaders reported difficulties in modifying the EHR, and only $57 \%$ of hospitals were able to integrate pathway content into the EHR by the end of the 15-month collaborative. This likely played a role in limiting the reach of electronic pathway orders to only $63 \%$ of children hospitalized with asthma. Difficulties in modifying EHRs have been reported as a barrier in prior qualitative studies of asthma pathway implementation (10), and have been associated with poor performance prior studies of pediatric learning collaboratives (24). EHRs can be leveraged to support pathway implementation in a number of ways, including providing clinical decision support for providers in the form of order sets, linking providers to more detailed evidence-based guidelines, enabling communication between providers, and automating data collection on the pathway implementation process (e.g.use of the pathway, clinical outcomes) (10). Central development of pediatric asthma order sets by commonly used EHR manufactures or streamlining of workflows needed to modify EHRs may help overcome this barrier. However, more research is needed to identify efficient ways to add functionality to EHRs and to understand how to optimize EHR modifications to improve quality of care.

Physician implementation leaders were satisfied with and valued pathway implementation. The benefit they most frequently reported was improvements in quality of care, including improvements in patient-centered outcomes (e.g.recovery time/length of hospital stay). Leaders also reported that improvements in care extended to children with other conditions than the condition of focus, asthma. This was because pathway content and educational materials (e.g.smoking cessation resource referral, respiratory scoring) sometimes applied to the care of children with other respiratory illnesses.
Another benefit of pathway implementation was enhanced interdisciplinary collaboration across providers and units of the hospital (e.g.emergency department, intensive care unit). These findings indicate that the benefits of inpatient pediatric asthma pathway implementation may extend far beyond the population of interest, potentially to all children cared for across many settings within a hospital.

Physician implementation leaders also highlighted the critical supports provided by the national learning collaborative. External development of the pathway toolkit and educational materials saved local implementation leaders' time. Leaders also reported that these resources were powerful in motivating practice change, because they were vetted by a reputable national organization (AAP). The collaborative also provided connections to peers at other hospitals that were engaged in pathway implementation. Implementation leaders reported that these connections facilitated sharing of implementation learnings. Thus, learning collaboratives may provide crucial supports for disseminating and implementing pathways and improving quality of care $(21,22,24)$. Indeed, the Value in Inpatient Pediatrics Network has seen growing demand for its national learning collaboratives, with a rise in participation from 17 hospitals to over 100 hospitals between 2008 and 2018 (13).

In this study, we applied an established implementation science framework (16) and used mixed methods to provide a global and detailed evaluation of the asthma pathway implementation process. We used a diverse, national sample of hospitals. However, this study has limitations. First, most data were gathered via self-report from physician implementation leaders only. Surveying other types of clinicians or patients/ family members may have yielded different perspectives and results. To our knowledge, patients and family members have not yet been included in pediatric asthma pathway research, thus, represent an important future area of study. However, these physician implementation leaders were the most knowledgeable regarding the pathway implementation process we sought to evaluate. In addition, some survey data was provided retrospectively and may be affected by recall bias. We were only able to verify implementation data at 4 hospitals, and this sample may not be generalizable to all participating sites. However, the subset of sites that were visited showed high validity of the survey responses (19 of 20 responses verified as correct). We defined reach as placement of a pathway/protocol order in a patient's chart, but this could not capture use of a paper pathway/protocol in the patient's care 
or use of other core pathway components. While this may underestimate the true "reach" of the intervention, it was the best marker of reach available via chart review and feasible to collect during this study. Lastly, our analysis was not able to determine which implementation practices, such as EHR integration, are most effective in improving quality of care.

\section{Conclusions}

Pathway implementation is both feasible and effective in improving care for children hospitalized with asthma across diverse hospital settings and should be prioritized by policymakers, hospital leaders, and clinicians. Important areas of future research include understanding the perspectives of patients and family members, identifying ways to improve the ease and effectiveness of EHR modifications, and determining how to ensure sustained improvements in care.

\section{Acknowledgements}

We would like to acknowledge AAP staff and all the local PIPA implementation leaders that made this study possible.

\section{Financial disclosures}

The authors have no financial relationships relevant to this article to disclose.

\section{Conflict of interest}

Dr. Cabana has consultancy positions with Biogaia AB, Novartis, and Phadia, and acts as a review panel member for Genentech. The other authors have indicated they have no potential conflicts of interest to disclose.

\section{Funding}

This study had no external funding. Drs. Kaiser and Parikh are supported by career development grants from the Agency for Healthcare Research and Quality (K08HS024592 and K08HS024554) but this agency played no role in the design, data collection, analysis, or reporting of this study.

\section{References}

1. Witt WP, Weiss AJ, Elixhauser A. Overview of hospital stays for children in the United States, 2012. Rockville, MD: Agency for Healthcare Research and Quality; 2014.

2. Centers for Disease Control. Asthma surveillance data 2017. 2019; https://www.cdc.gov/asthma/asthmadata. htm. Accessed July 1, 2019.
3. Dayal A, Alvarez F. The effect of implementation of standardized, evidence-based order sets on efficiency and quality measures for pediatric respiratory illnesses in a community hospital. Hosp Pediatr. 2015;5(12): 624-9. doi:10.1542/hpeds.2015-0140.

4. Kaiser SV, Rodean J, Bekmezian A, Hall M, Shah SS, Mahant S, Parikh K, Auerbach AD, Morse R, Puls $\mathrm{HT}$, et al. Effectiveness of pediatric asthma pathways for hospitalized children: a multicenter, national analysis. J Pediatr. 2018;197:165-71 e162. doi:10.1016/j. jpeds.2018.01.084.

5. Chen KH, Chen CC, Liu HE, Tzeng PC, Glasziou PP. Effectiveness of paediatric asthma clinical pathways: a narrative systematic review. J Asthma. 2014;51(5): 480-92. doi:10.3109/02770903.2014.887728.

6. Edwards E, Fox K. A retrospective study evaluating the effectiveness of an asthma clinical pathway in pediatric inpatient practice. J Pediatr Pharmacol Ther. 2008;13(4):233-41. doi:10.5863/1551-6776-13.4.233.

7. Nkoy F, Fassl B, Stone B, Uchida DA, Johnson J, Reynolds C, Valentine K, Koopmeiners K, Kim EH, Savitz L, et al. Improving pediatric asthma care and outcomes across multiple hospitals. Pediatrics. 2015; 136(6):e1602-1610. doi:10.1542/peds.2015-0285.

8. Nkoy F, Fb Stone B, Uchida D, Johnson J, Ryenolds C, Valentine K, Koopmeiners K, Unsicker Eh Savitz L, Maloney C. Impact of an evidence-based care process model implementation on childhood asthma readmission disparities. Paper presented at: Pediatric Academic Societies National Conference; 2016; Balitmore MD.

9. Wazeka A, Valacer DJ, Cooper M, Caplan DW, DiMaio M. Impact of a pediatric asthma clinical pathway on hospital cost and length of stay. Pediatr Pulmonol. 2001;32(3):211-6. doi:10.1002/ppul.1110.

10. Kaiser SV, Lam R, Cabana MD, Bekmezian A, Bardach NS, Auerbach A, Rehm RS. Best practices in implementing inpatient pediatric asthma pathways: a qualitative study. J Asthma. 2019;1-11. doi:10.1080/ 02770903.2019.1606237.

11. Pound CM, Gelt V, Akiki S, Eady K, Moreau K, Momoli F, Murchison B, Zemek R, Mulholland B, Kovesi $\mathrm{T}$, et al. Nurse-Driven clinical pathway for inpatient asthma: a randomized controlled trial. Hosp Pediatr. 2017;7(4):204-13. doi:10.1542/hpeds.2016-0150.

12. Kaiser SV, Jennings B, Rodean J, et al. Pathways for Improving Inpatient Pediatric Asthma Care (PIPA): a multi-center, national study. Pediatrics. 2020; In Press.

13. American Academy of Pediatrics. Value in inpatient pediatrics network 2019; https://www.aap.org/en-us/ professional-resources/quality-improvement/Pages/Valuein-Inpatient-Pediatrics.aspx. Accessed August 1, 2019.

14. Value in Inpatient Pediatrics Network. PIPA (Pathways for Improving Pediatric Asthma Care): inpatient pathway toolkit. 2019. https://downloads. aap.org/DOCCSA/PIPAInpatientToolkit.pdf. Accessed AUgust 1, 2019.

15. Institute for Healthcare Improvement. The breakthrough series: IHI's collaborative model for achieving breakthrough improvement. IHI innovation series white paper. Boston; 2003. 
16. Glasgow RE, Vogt TM, Boles SM. Evaluating the public health impact of health promotion interventions: the RE-AIM framework. Am J Public Health. 1999; 89(9):1322-7. doi:10.2105/AJPH.89.9.1322.

17. Lion KC, Wright DR, Spencer S, Zhou C, Del Beccaro M, Mangione-Smith R. Standardized clinical pathways for hospitalized children and outcomes. Pediatrics. 2016;137(4):e20151202-e20151202. doi:10.1542/peds. 2015-1202.

18. American Hospital Association. AHA annual survey database. 2019. https://www.ahadata.com/aha-annualsurvey-database-asdb/. Accessed November 10, 2017.

19. Helfrich CD, Li YF, Sharp ND, Sales AE. Organizational readiness to change assessment (ORCA): development of an instrument based on the Promoting Action on Research in Health Services (PARIHS) framework. Implementation Sci. 2009;4(1): 38. doi:10.1186/1748-5908-4-38.

20. Centers for Medicare and Medicaid Services. Medicaid eligibility. 2020; medicaid.gov/medicaid/eligibility/index.html. Accessed February 1, 2020.
21. Parikh K, Biondi E, Nazif J, Wasif F, Williams DJ, Nichols E, Ralston S. A Multicenter collaborative to improve care of community acquired pneumonia in hospitalized children. Pediatrics. 2017;139(3): e20161411. doi:10.1542/peds.2016-1411.

22. Ralston SL, Garber MD, Rice-Conboy E, Mussman GM, Shadman KA, Walley SC, Nichols E. A multicenter collaborative to reduce unnecessary care in inpatient bronchiolitis. Pediatrics. 2016;137(1): e20150851. doi:10.1542/peds.2015-0851.

23. McDaniel CE, Jennings $R$, Schroeder AR, Paciorkowski N, Hofmann M, Leyenaar J. Aligning inpatient pediatric research with settings of care: a call to action. Pediatrics. 2019;143(5):e20182648. doi: 10.1542/peds.2018-2648.

24. Ralston SL, Atwood EC, Garber MD, Holmes AV. What works to reduce unnecessary care for bronchiolitis? A qualitative analysis of a national collaborative. Acad Pediatr. 2017;17(2):198-204. doi:10.1016/j.acap. 2016.07.001. 